\title{
BARYONS 2002: OUTLOOK
}

\author{
WOLFRAM WEISE \\ ECT* , Villa Tambosi, I-38050 Villazzano (Trento), Italy \\ and \\ Physik-Department, Technische Universität München, \\ D-85747 Garching, Germany
}

\section{Preamble}

This is a sad moment: the outlook concluding this conference should have been presented by Nathan Isgur. It will be difficult to meet his standards.

In his introductory talk at BARYONS '98 in Bonn, Nathan drew a picture of his vision for "Strong QCD". The zeroth order starting point, he hypothesized, for building hadrons should be relativistic constituent quarks tied together by flux-tube gluon dynamics. In a book-keeping strategy organized in terms of inverse powers of $N_{c}$, the number of colors, one should then add the quark-antiquark sea and other $1 / N_{c}$ effects as perturbations. We were also reminded of the potentially important role played by the $q \bar{q}$ vacuum condensate in connecting valence quarks and potentials on one hand with the current quarks and gluons of QCD on the other.

All of these key notions have been very visible again at the present conference. So, have we advanced substantially in our understanding of constituent quarks, gluonic flux tubes, confinement, spontaneous chiral symmetry breaking and the QCD vacuum since our last BARYONS meeting? I believe the answer is a forceful "yes", in view of the significant progress achieved by the joint efforts of experiments and theory, but many issues still remain to be resolved.

\section{Lattice QCD}

With steadily increasing computational power, "solving" QCD on large Euclidean lattices has now become an important part of hadron physics. Lattice QCD was addressed by no less than four plenary speakers (C. Davies, R. Edwards, G. Schierholz, A.W. Thomas) who gave impressive surveys of the progress made in recent years. The gluonic flux tube and its translation into a static confining potential between (infinitely heavy) color sources has become "reality" on the lattice. The Y-shaped potential characteristic of static three-quark systems is predicted by lattice QCD, as well as hybrid

outlook: submitted to World Scientific on November 6, 2018 
baryons with three heavy quarks coupled to gluonic excitations (reviewed by $\mathrm{Ph}$. Page). But so far, the dynamics of light quarks is not under control, at least not in the context of the confinement problem.

New developments have been reported on baryon observables (masses, electromagnetic and axial form factors, moments of structure functions) extracted from lattice QCD. Improved actions are designed to reduce discretization errors. Effects of the finite lattice volume are seriously addressed. Procedures leading beyond the "quenched" (no-fermion-loops) approximation are well on their way.

Still, these results are limited to relatively large quark masses. The typical "light" quark masses manageable on the lattice up to now, are 10-20 times larger than the $\mathrm{u}$ - and d-quark masses determined at renormalization scales around $1 \mathrm{GeV}$. The corresponding pion masses are well above $0.5 \mathrm{GeV}$, far away from physical reality, so that important aspects of chiral pion dynamics are presumably suppressed.

While lattice calculations using small quark masses close to the chiral limit are out of reach in the foreseeable future, such huge efforts may not even be necessary. Low-energy QCD in the light-quark sector is realized in the form of an effective field theory based on chiral symmetry, and this theory can be used efficiently to extrapolate between lattice results obtained at higher u- and d-quark masses and the "real world" of small quark masses. The feasibility of such extrapolations employing suitable Padé approximants (Leinweber, Melnitchouk, et al.) or extended versions of chiral perturbation theory (Hemmert et al.) has been discussed at this conference, with promising results. Once the lattice calculations will approach pion masses around $300 \mathrm{MeV}$ in the near future, reliable extrapolations using effective field theory methods have a good chance of closing the remaining gap between lattice QCD and actual observables.

\section{Constituent Quarks}

What is a constituent quark? Over several decades we have learned to live with this phenomenological concept without really understanding what it means in detail. Constituent quarks have been remarkably successful in describing ratios of baryon magnetic moments and organizing the symmetry breaking patterns seen in the hadron spectrum. One can think of constituent quarks as quasi-particles in a way analogous to those introduced in many-body problems. For example, a good approximation for an interacting electron gas at moderate densities is in terms of weakly interacting quasi-electrons, i. e. electrons with their Coulomb interactions screened by a cloud of electron-

outlook: submitted to World Scientific on November 6, 2018 
hole excitations. Similarly, constituent quarks might be viewed as quarks dressed by clouds of quark-antiquark pairs and gluons. In distinction from the behaviour commonly associated with quasi-particles, however, constituent quarks do not interact weakly: they experience color confinement, and their residual interactions must be strong enough to generate the observed large hyperfine splittings in the baryon spectrum.

One can address the constituent quark question from another perspective, by asking: how many quarks are there in a baryon?

The answer from spectroscopy would simply be: $N=3$. This is, of course, the basis of the time-honoured Isgur-Karl model, and we have heard an updated review on its further developments, successes and limitations by S. Capstick. From a different viewpoint, E. Klempt reminded us of the important role played by the color degree of freedom beyond just antisymmetrizing the baryon wave functions.

When looking at deep-inelastic lepton-nucleon scattering the answer to the same question would rather be: $N \rightarrow \infty$. This is the picture projected by the HERA data and reviewed by R. Yoshida. Counting quarks means, in this context, taking the integral of $F_{2}(x) / x$ over the Bjorken scaling variable $x$, or equivalently, $\int d \ln x F_{2}(x) \rightarrow \infty$, given the observed behaviour of the structure function $F_{2}(x)$ at small $x$. (Of course one must recall that small$x$ physics, when viewed in the laboratory frame, involves quark-antiquark and gluon fluctuations of the high-energy virtual photon which extend over distances large compared to the proton size. The fact that $N \rightarrow \infty$ is therefore a statement about the interacting photon-nucleon system at high energies, not about the isolated nucleon.)

Constituent quarks somehow interpolate between "three" and "infinity". It is likely that their properties are linked to the rich, highly non-trivial structure of the QCD vacuum. Y. Simonov pointed out that constituent quark masses and magnetic moments might have a direct relationship to the gluonic string tension. D. Diakonov emphasized the importance of instantons, large fluctuations of the gluon field. In this approach, the constituent quark mass at zero momentum is proportional to the average instanton size and inversely proportional to the squared average instanton separation in the QCD vacuum. With an instanton radius of about $1 / 3 \mathrm{fm}$ and a separation of $1 \mathrm{fm}$, compatible with instanton simulations in lattice QCD, the zero-momentum constituent quark mass emerges at about $350 \mathrm{MeV}$, a remarkable result. The "running" quark mass decreases rapidly at momentum scales larger than $1 \mathrm{GeV}$, just as it should. Lattice QCD studies of the Euclidean quark propagator performed by the Adelaide group (D. Leinweber et al.) find a similar behaviour of the momentum dependent quark mass in the Landau gauge (with the caveat to

outlook: submitted to World Scientific on November 6, 2018 
be added that a constituent quark mass is not a gauge invariant quantity in QCD).

Confinement still persists as the primary challenge in all efforts trying to understand the physical meaning of constituent quarks. Confinement is missing, for example, in the instanton approach despite its apparent successes.

The nature of residual interactions between constituent quarks has been a much debated theme. Spin-flavour correlations characteristic of Goldstone boson exchange (as discussed e. g. by L. Glozman et al.) have been held against traditional one-gluon exchange descriptions. There is good reason to expect that both kinds of forces act (amongst others) peacefully side by side. One should not forget, after all, that a local interaction between two color-currents generates, via Fierz transformation, all sorts of exchange terms including color-singlet spin-flavour exchanges with meson quantum numbers.

\section{Baryon Resonances}

The research on baryon resonances has progressed enormously during the time span between this and the last BARYONS conference. These new developments, primarily driven by the experimental programmes of the CLAS detector at JLab, at Mainz and Bonn and at the GRAAL and LEGS facilities, define a new level of high-precision measurements. Out of the many highlights, only a few can be discussed here, with no attempt to achieve even partial completeness.

An impressive example is the multipole analysis of neutral pion electroproduction data, reported by V. Burkert, which sets new standards for the separation of magnetic dipole and electric quadrupole transition formfactors in the delta resonance region at momentum transfers as high as $1 \mathrm{GeV}$. The search for missing resonances in the baryon spectrum is entering a new era as well. CLAS data on two-pion electroproduction indicate signals in the spectrum around center-of-mass energies of $1.7 \mathrm{GeV}$, which are not covered by commonly used isobar models. Virtual Compton scattering ( $e p \rightarrow e p \gamma)$ with its minimal final state interaction also proves to be a valuable source of information for systematic baryon resonance studies. At JLab's Hall A the first measurement covering the entire region up to $W=2 \mathrm{GeV}$ has been performed (reported by H. Fonvieille) and displays strong resonance excitations. Very impressive results have also been presented for resonance searches in electroproduction on the proton leading to kaon-hyperon final states, again by a CLAS collaboration. To round off the picture, T. Nakano showed promising developments in kaon photoproduction at SPRING-8 with focus on the $\Lambda(1405)$ and $\Lambda(1520)$, the detailed nature of which is still a matter of debate

outlook: submitted to World Scientific on November 6, 2018 
between standard quark models and coupled channels approaches.

New high quality data from GRAAL were reported by A. D'Angelo. Following previous pioneering measurements at Mainz and Bonn, eta meson photoproduction now covers the region from near-threshold to $1.1 \mathrm{GeV}$ in a single experiment, with stunning accuracy and the quest for further explorations into a possible structure around a photon energy of $1.05 \mathrm{GeV}$. Another very interesting case is the photon beam asymmetry measured in omega meson production on the proton at photon energies above $1 \mathrm{GeV}$. Purely diffractive $t$-channel exchange mechanisms would not produce any asymmetry at all. Non-zero asymmetry signals measured with high precision are therefore a distinct testing ground for $N^{*}$ resonance studies.

Polarisation observables are a key to the detailed analysis and understanding of resonances in multipole amplitudes. Experiments with polarized photon beams on polarized protons performed at Mainz and Bonn, and also recently at LEGS (as summarized by A. D'Angelo), are paving the way for an accurate examination of the Gerasimov-Drell-Hearn sum rule. The relevant GDH integral when taken up to about $1.8 \mathrm{GeV}$ seems to pass beyond the canonical sum rule value by about $5-10 \%$. A further extension of the double polarization measurements to higher energies is therefore mandatory.

The theoretical state of the art on baryon resonance physics was reviewed by T. Sato. Various models exist to deal with the multipole amplitudes for photo- and electroproduction, mostly based on effective Lagrangians with inclusion of dominant baryon resonances. The situation can be summarized as follows. The physics of the delta resonance is quite well under control. An exception is the $N \rightarrow \Delta$ quadrupole transition amplitudes for which the detailed understanding still needs to be improved. While models including pion cloud effects are in good agreement with JLab data for $Q^{2}$ above $0.4 \mathrm{GeV}^{2}$, A. Bernstein pointed out in discussions that such calculations fail to reproduce the accurate Bates data at $Q^{2} \simeq 0.1 \mathrm{GeV}^{2}$ where the theoretical treatment of pion cloud effects should actually be more reliable. In the second resonance region it is important to improve on descriptions of channels with two pions in the continuum. The theory of such $\pi \pi N$ three-body channels has not yet reached a quantitatively satisfactory level. In the third and higher resonance region there is still much work to do on the theoretical side, even conceptually, concerning detailed resonance versus background analysis. Problems of this sort, with quasi-bound states embedded in a multiparticle continuum, exist also in nuclear physics, atomic physics and quantum optics, and it may be useful to borrow from the reaction theory expertise developed in those fields.

Last not least, with the high productivity of several electron and photon facilities now in operation and further upgrades in sight, one should not forget

outlook: submitted to World Scientific on November 6, 2018 
the important information provided by experiments with hadron beams, as pointed out by M. Sadler. The picture will not be complete unless electromagnetic and hadronic probes are systematically used in a complementary way.

\section{Spin Structure}

Investigations of the nucleon's spin structure are entering a new phase. It is now well established that quarks carry only a fraction, less than one third, of the proton spin. The next challenges are: to extract the flavour decomposition of those spin fractions, to isolate the gluon contribution to the total spin, and to analyse transverse spin degrees of freedom which provide additional independent pieces of information.

G. van der Steenhoven gave an impressive status report and an exciting outlook into coming years. The HERMES experiment continues improving the data quality for the polarized structure functions $g_{1}$ of the proton, the deuteron, and of the neutron as deduced from ${ }^{3} \mathrm{He}$. The accuracy of the flavour decomposition of quark spin fractions from the analysis of semiinclusive deep-inelastic scattering is expected to improve as well. The gluon contribution to the proton spin will be explored with COMPASS, HERMES and RHIC, by photon-gluon fusion and reconstruction of the produced charmed quark-antiquark pairs, and by measuring tracks of high transverse momentum, with an expected accuracy at the $10 \%$ level. A novel quantity of considerable current interest is the density of transversely polarized quarks inside a transversely polarized proton, the "transversity" distribution $h_{1}$. By its very nature, $h_{1}$ emphasizes more prominently the valence quark aspects of spin structure, so it yields important complementary information to the longitudinal quark spin distributions.

A further new dimension opens through Deep Virtual Compton Scattering (DVCS) measurements and the extraction of Generalized Parton Distributions (summarized by M. Diehl). According to Ji's sum rule, DVCS has access to the total quark angular momentum (its spin plus orbital angular momentum). The feasibility of DVCS experiments has recently been demonstrated, in parallel, both by HERMES and by the CLAS collaboration at JLab.

Another important issue is the $Q^{2}$ evolution of spin structure functions and the influence of baryon resonances at smaller $Q^{2}$. JLab has taken a leadership role in these projects, as reviewed by R. de Vita. The preliminary data on the first moment of $g_{1}$ for the proton begin to provide the systematics that is necessary to interpolate between the deep inelastic scattering region and the non-perturbative spin physics at lower $Q^{2}$, down to the GDH sum

outlook: submitted to World Scientific on November 6, 2018 
rule at $Q^{2}=0$. A very interesting new result is the CLAS measurement of $g_{1}(x)$ for the proton at $Q \simeq 1 \mathrm{GeV}$. It demonstrates the importance of the delta resonance (and possibly higher resonances) in the nucleon spin structure problem, even at reasonably large $Q$. This behaviour was predicted several years ago (Edelmann et al.) and the theory has been further developed in the meantime (Simula et al.). To those who confidently expected perturbative QCD to start already at $Q \sim 1 \mathrm{GeV}$, this came unexpected. It is an interesting question now whether the basic idea of quark-hadron duality (reviewed by S. Jeschonnek at the conference), namely that properly averaged hadronic observables can be described in terms of perturbative QCD, is at work also for spin structure functions.

\section{$6 \quad$ Nucleon Form Factors and Polarizabilities}

A bright highlight in the exploration of nucleon electromagnetic form factors (reviewed by E. Brash) is the recent observation by a polarization transfer measurement at JLab's Hall A that charge and magnetization distributions in the proton are not proportional as was long thought. The ratio $\mu G_{E} / G_{M}$ drops continuously down to almost one half at $Q^{2} \simeq 4 \mathrm{GeV}^{2}$, whereas it was previously assumed to be equal to one on the basis of simple universal dipole parametrizations for those form factors. The JLab data have triggered a reexamination of the Rosenbluth extraction of form factors from earlier cross section measurements. Some relativistic constituent quark models and the cloudy bag model describe the new situation properly, at least at a qualitative level. Further thinking about the dynamics which governs the proton's constituents and their wave functions will obviously be stimulated by these new developments.

Parity violation in elastic electron scattering as a tool to investigate admixtures of strange quark-antiquark pairs in the nucleon has been reviewed by F. Maas. Earlier predictions of large ground state matrix elements for strange quark currents in the nucleon are not confirmed. The SAMPLE experiment at Bates, the HAPPEX measurements at JLab and recent advances at Mainz all set quite low limits for the strange vector current in the proton, considerably lower than the $10 \%$ level expected from previous estimates. This does not imply, of course, that strangeness is equally unimportant in other types of currents. For example, the scalar density of strange quarks in the nucleon might be considerably more pronounced. Renewed interest in this question is triggered by recent claims that the sigma term of the nucleon is substantially larger than its previously deduced value of $(45 \pm 8) \mathrm{MeV}$.

Electromagnetic and spin polarizabilities have always played an important

outlook: submitted to World Scientific on November 6, 2018 
part in our understanding of nucleon structure. Virtual Compton Scattering (VCS, reviewed by H. Fonvieille) and the generalized polarizabilities deduced from such experiments will be a rich source of new insights into the low-energy dynamics of the nucleon's internal degrees of freedom. The accuracy reached in the pioneering VCS measurements at Mainz is a major step forward, and together with the VCS programmes at JLab and Bates, the progress in the field will be significant. On the theoretical side, the analysis of VCS data is greatly helped by sophisticated dispersion relation methods (B. Pasquini et al.) and by effective field theory approaches (H. Grießhammer et al.).

\section{Chiral Dynamics}

Low-energy QCD with light $u$ - and $d$-quarks is realized in the form of an effective field theory of pions, the Goldstone bosons of spontaneously broken chiral symmetry, coupled to "heavy" sources such as baryons. The low-energy expansion of this theory in small momenta or small quark masses is called chiral perturbation theory $(\mathrm{ChPT})$. Both its predictive capacity and its inherent limitations were lucidly summarized by T. Becher. Based on the pioneering work by Gasser and Leutwyler and by Weinberg, ChPT has been a remarkably successful theoretical tool, not only in its traditional domain, threshold pion-pion scattering, but also in understanding the role of the nucleon's pion cloud in pion-nucleon scattering and photoproduction processes.

One of those traditionally successful examples has been neutral pion photoproduction on the proton close to threshold. A further challenging test for ChPT is $\pi^{0}$ production induced by virtual photons and its systematic $Q^{2}$ dependence. H. Merkel reported on the very accurate Mainz measurements at $Q^{2}=0.05$ and $0.1 \mathrm{GeV}^{2}$, close to threshold, a region where the chiral lowenergy expansion is expected to work well. But apparently it doesn't! The ChPT predictions of V. Bernard et al. systematically fail as one moves away from the real-photon point to $Q^{2}>0$. (Admittedly, the MAID multipole amplitudes have similar difficulties when confronted with these high-precision data.) The issue raised by these findings is twofold: the data systematics should be enlarged by measurements at several additional values of $Q^{2}$; and the ChPT calculations will have to meet this challenge by moving to the next higher order.

Rigorous ChPT is limited in its applicability to sometimes very small convergence regimes when resonances are produced nearby. A prominent example is the delta resonance. In the "official" power counting philosophy, its effects are relegated to higher orders. But the prominent role of the $\Delta(1232)$ in the strong $M 1$ transition that governs the paramagnetic response of the

outlook: submitted to World Scientific on November 6, 2018 
nucleon, an evident empirical fact, calls for a different kind of book-keeping. In the large $N_{C}$ limit, the nucleon and the $\Delta$ are degenerate: there is no particular reason to treat the baryon octet and the decuplet on a different basis in heavy-baryon ChPT. Extended versions of chiral effective field theory do in fact promote the delta to leading order in combined chiral and large- $N_{C}$ expansions, a useful strategy. Such a scheme has been successfully employed, for example, in analysing the strong energy dependence of the generalized magnetic dipole polarizability of the nucleon (as reported by H. Grießhammer). It is also a key element in chiral extrapolations of nucleon properties deduced from lattice QCD (T. Hemmert et al., W. Melnitchouk et al.).

With inclusion of strangeness, non-perturbative features of meson-baryon dynamics can be handled efficiently by combining the chiral SU(3) chiral effective Lagrangian with coupled channels techniques familiar from nuclear reaction theory. While sacrificing some of the puristic ChPT power counting rules, the physics benefit from iterating important subclasses of driving amplitudes to all orders is certainly rewarding. Most recent developments (E. Kolomeitsev, M. Lutz), reported at this conference, now include the full baryon decuplet in addition to the octet. The number of free parameters is constrained by large- $N_{C}$ bookkeeping. The quantitative agreement of such calculations with a large number of observables in a variety of meson-baryon channels is quite remarkable.

Is the restoration of chiral symmetry (from its spontaneously broken Nambu-Goldstone realization to the unbroken Wigner-Weyl mode) visible in the high-mass sector of the baryon spectrum? This interesting question (raised by L. Glozman and T. Cohen) alludes to possible parity dublets in the spectrum, i. e. degeneracies between positive and negative parity baryon resonances of the same spin. Several such cases can be located in the mass region around $2 \mathrm{GeV}$, but there are several other examples which seem not to follow this rule. The parity dublet criterion in its strict sense would apply to narrow states, whereas most of the excited baryon states in question have large widths. On the other hand, in the asymptotic continuum region, identical spectral distributions of parity partners are a familiar phenomenon, as seen for example in the large $s$ behaviour of vector and axial vector spectral functions in the $\rho$ and $a_{1}$ channels. In any case, such discussions emphasize again the need for more systematic explorations of high lying excitations in the $N$ and $\Delta$ spectrum.

outlook: submitted to World Scientific on November 6, 2018 


\section{Looking forward}

This conference has given us once again a very lively and convincing demonstration that baryons (and their prototype, the nucleon) represent a most fascinating QCD many-body problem. It exhibits all of the phenomena associated with QCD, notably confinement, spontaneous chiral symmetry breaking and asymptotic freedom. While the perturbative high-energy sector of QCD is quite well understood, its non-perturbative features, the complexity of the QCD vacuum and its relationship to confinement and spontaneous symmetry breaking continue to be outstanding challenges. Last but not least, nuclear physics needs ultimately to be understood in these terms, and the issue (alluded to in G. A. Miller's talk) of how a nucleon changes its properties in a nuclear environment (a QCD many-body problem intertwined with the nuclear many-body problem) is as burning as ever.

One can say without exaggeration that the field is in a promising and forward-looking situation. It is driven by three basic elements which are now operating in a healthy balance: it is "data driven", with emphasis on high precision and full utilization of polarization observables; it is "brain driven", with advanced theoretical approaches constrained by symmetries of QCD; and it is "computer driven", with future computational capacity and speed progressing into the multi Teraflop regime.

Given the variety of running facilities at our disposal and upgrades in sight (JLab, Bates, MAMI C, HERMES, COMPASS, RHIC-Spin, SPRING$8, \ldots$, amongst others), the perspectives for deepening our understanding of QCD phenomena are certainly good. But one should also re-emphasize the importance of systematic investigations using hadron beams. In particular, our knowledge about systems with strange and charmed quarks is still underdeveloped.

I close with an expression of deep gratitude to Bernhard Mecking, John Domingo and all members of the JLab team who have made this conference an exciting and memorable event.

outlook: submitted to World Scientific on November 6, 2018 